\title{
Vom Anfang und Ende des Kapitalismus
}

Ulrike Herrmann

Der Kapitalismus ist ein Rätsel. Er hat längst alle Lebensbereiche durchdrungen, aber seine Deutung ist noch immer umstritten. Das beginnt schon beim Begriff: Das Wort Kapitalismus zu verwenden, gilt in Deutschland häufig als slinks` oder gar >marxistisch . In den USA hingegen wird der Ausdruck Kapitalismus völlig selbstverständlich benutzt, der im Übrigen auch gar nicht von Karl Marx stammt. ${ }^{1}$

Der Begriff Kapitalismus hat den Vorteil, dass er präzise beschreibt, was die heutige Wirtschaftsform auszeichnet: Es geht um den Einsatz von Kapital mit dem Ziel, hinterher noch mehr Kapital zu besitzen, also einen Gewinn zu erzielen. Es handelt sich um einen Prozess, der exponentielles Wachstum erzeugt. ${ }^{2}$

Aber was ist dieses >Kapitalk? Es ist nämlich nicht das Gleiche wie Geld, obwohl der Begriff im Alltag oft synonym verwendet wird. Geld gab es schon immer, es ist mindestens 4000 Jahre alt: Die ersten Texte der Menschheit stammen aus Mesopotamien und wurden nicht etwa verfasst, um Literatur zu überliefern - sondern um Zahlungsverpflichtungen zu verzeichnen.

Während das Geld uralt ist, ist das Kapital noch jung. Der moderne Kapitalismus ist etwa 1760 im Nordwesten Englands entstanden, als Textilfabrikanten Webstühle und Spinnereien mechanisierten. Heute wirken diese Maschinen klein und zierlich, aber mit ihnen begann eine neue Epoche. Erstmals in der Geschichte wurde die menschliche Arbeitskraft systematisch durch Technik ersetzt, und damit stieg das Einkommen deutlich. Seit Jahrtausenden hatte die Wirtschaft weitgehend stagniert, aber nun wuchs sie exponentiell. Das `Kapital im Kapitalismus ist also nicht das Geld, sondern es sind die effizienten Produktionsprozesse und der technische Fortschritt.

Der Begriff Kapitalismus wurde erst Ende des 19. Jahrhunderts gebräuchlich und setzte sich in Deutschland spätestens mit Werner Sombarts Hauptwerk Der moderne Kapitalismus (1902) durch, vgl. Sombart, Werner: Der moderne Kapitalismus. Erster Band: Die Cenesis des Kapitalismus, Leipzig: Duncker \& Humblot 1902.

2 Diese grobe Definition lässt sich natürlich beliebig verfeinern. Schon 1918 wurden in der damaligen Wirtschaftsliteratur 111 verschiedene Definitionen von Kapitalismus gefunden, vgl. Osterhammel, Jürgen: Die Verwandlung der Welt. Eine Geschichte des 19. Jahrhunderts, München: C.H. Beck 2011, S. 953. 
Es war eine Revolution, kein Mehr vom Gleichen. Der österreichische Ökonom Joseph Schumpeter hat für diesen fundamentalen Wandel ein prägnantes Bild gefunden: »Man kann beliebig viele Postkutschen aneinanderreihen - und trotzdem wird daraus niemals eine Eisenbahn. $\ll^{3}$

Doch warum hat die Industrialisierung ausgerechnet in England eingesetzt? Und warum ab 1760? »Obwohl tausende von Büchern geschrieben wurden, bleibt es ein gewisses Rätsel«, konstatiert die amerikanische Wirtschaftshistorikerin Joyce Appleby. ${ }^{4}$ Technologisch waren die Briten nämlich nicht besonders avanciert und wussten anfangs auch nicht mehr als die antiken Römer. Die Dampfmaschine beruhte auf Prinzipien, die seit Archimedes bekannt waren.

Die Wirtschaftshistoriker/-innen haben inzwischen weit mehr als zwanzig verschiedene Theorien entwickelt, um zu erklären, warum der moderne Kapitalismus in England seinen Anfang nahm. ${ }^{5}$ Die überzeugendste Analyse setzt bei den Produktionskosten an: Die englischen Löhne waren im 18. Jahrhundert die höchsten der Welt - so dass die britischen Waren international nicht mehr konkurrenzfähig waren. Nur weil die Menschen teuer waren, lohnte es sich erstmals, Maschinen einzusetzen und mit der Industrialisierung zu beginnen. ${ }^{6}$

Die britische Erfahrung ist noch immer aktuell: Der Kapitalismus entwickelt sich nur stabil, solange die Reallöhne steigen und mit der zunehmenden Effizienz mithalten. Viele Unternehmer wollen es nicht glauben, aber hohe - nicht niedrige - Gehälter fördern das Wachstum und machen die Firmen reich.

Obwohl der Kapitalismus nun rund 250 Jahre alt ist, halten sich noch immer hartnäckige Missverständnisse. Dazu zählt der Glaube, dass der Kapitalismus das Gleiche wie eine >Marktwirtschaft< sei. Doch die Marktwirtschaft gibt es nicht, oder nur in kleinen Nischen.

Bereits ein Blick in die Geschichte zeigt, dass der moderne Kapitalismus mehr sein muss als nur eine Ansammlung von Märkten. Märkte existierten bereits in der griechischen Antike vor 2500 Jahren. Auch die Araber hatten ihre Souks, Türken und Perser ihre Basare. Inder und Chinesen tauschten ihre Waren ebenfalls auf Märkten aus, aber ein moderner Kapitalismus ist daraus nirgends entstanden.

Allerdings meint die Theorie von der Marktwirtschaft mehr, als dass nur Märkte vorhanden seien. Sie will vor allem beschreiben, wie faire Preise entstehen -

Schumpeter, Joseph A.: The Theory of Economic Development. An Inquiry into Profits, Capital, Credit, Interest and the Business Cycle, übersetzt von Redvers Opie, New Brunswick/London: Transaction Publishers 1983, S. 64.

4 Appleby, Joyce: The Relentless Revolution. A History of Capitalism, New York: Norton 2010, S. 10, eigene Übersetzung.

5 Vgl. Vries, Peer: Escaping Poverty. The Origins of Modern Economic Growth, Wien: Vienna University Press/V\&R unipress 2013.

6 Für eine umfassende Darstellung vgl. Allen, Robert C.: Clobal Economic History. A Very Short Introduction, Oxford u.a.: Oxford University Press 2011. 
nämlich durch umfassenden Wettbewerb. Viele Anbieter sollen auf viele Nachfrager treffen, so dass diese Konkurrenz sicherstellt, dass weder Firmen noch Kunden übervorteilt werden.

Diese attraktive Theorie hat jedoch einen Nachteil: Den unterstellten Wettbewerb gibt es höchstens eingeschränkt. Stattdessen wird unsere Wirtschaft von Großkonzernen geprägt, die von den Rohstoffen bis zum Absatz die gesamte Wertschöpfungskette kontrollieren. Bereits eine trockene Zahl des Statistischen Bundesamts sagt alles: Die bundesdeutsche Wirtschaft ist inzwischen extrem konzentriert, wie aus dem neuesten Statistischen Jahrbuch hervorgeht. Die Großkonzerne machen nur 0,7 Prozent aller Firmen in Deutschland aus - aber sie kontrollieren 66,7 Prozent des Umsatzes. ${ }^{7}$ Diese extreme Konzentration wirtschaftlicher Macht ist übrigens auch in allen anderen westlichen Ländern festzustellen.

Echte Marktwirtschaft gibt es zwar, aber sie findet meist in Nischen statt. Es sind die kleinen Selbstständigen, die sich im gnadenlosen Wettbewerb behaupten müssen. Ob Handwerker/-innen, Friseur/-innen, Gastwirt/-innen, Architekt/innen, kleine Ladenbesitzer/-innen oder die Betreiber/-innen einer Reinigung sie alle müssen sich der Konkurrenz stellen. Wenn das Essen nicht schmeckt, gehen die Kund/-innen beim nächsten Mal in ein anderes Restaurant. Kleine Firmen gibt es viele, doch dort findet nur ein Bruchteil der eigentlichen Wertschöpfung statt. Dominiert wird die Wirtschaft von wenigen Großkonzernen, die sich dem Wettbewerb weitgehend entziehen können.

Diese Entwicklung ist keineswegs neu, sondern war schon im 19. Jahrhundert zu beobachten. Allein zwischen 1879 und 1886 dürften rund 90 Kartelle in Deutschland entstanden sein, die meisten davon waren Preiskartelle. ${ }^{8}$ Aus der Sicht der Firmen war dies rational und sogar zwingend: Mit dem steigenden Einsatz von Technik nahmen die Investitionskosten ständig zu, und daher wollten die Unternehmen sicherstellen, dass Absatz und Preise hinterher stimmten und nicht durch schrankenlosen Wettbewerb ruiniert wurden. Damals zeigte sich erstmals ein Paradox, das den Kapitalismus bis heute prägt: Nur wenn das Risiko weitgehend ausgeschlossen ist, werden hohe Investitionen riskiert.

Exemplarisch ist die Geschichte der deutschen Elektroindustrie: 1882 begann der internationale Siegeszug der Glühbirne, die der US-Amerikaner Thomas Alva Edison erfunden hatte. In Deutschland gründete Emil Rathenau schon 1883 die Deutsche Edison-Gesellschaft für angewandte Elektrizität. Um jeden Ärger zu vermeiden, einigte er sich bereits vorab mit dem einzig denkbaren Konkurrenten mit der Firma Siemens \& Halske, die seit den 1860er Jahren Dynamomaschinen baute. Als Arrangement schlug Rathenau vor, dass er sämtliche Vorprodukte von

7 Vgl. Statistisches Bundesamt (Destatis): Statistisches Jahrbuch 2018, S. 526.

8 Vgl. Ziegler, Dieter: Die industrielle Revolution, 3., bibliographisch aktualisierte Auflage, Darmstadt: Wissenschaftliche Buchgesellschaft 2009, S. 108ff. 
Siemens beziehen würde - aber die Glühbirnen wollte er selbst herstellen. ${ }^{9}$ Der Markt wurde also schon verteilt, noch bevor er existierte.

Technische Entwicklungen machten diesen Glühbirnen-Vertrag zwar bald überflüssig, aber die gedeihliche Zusammenarbeit blieb. Als Emil Rathenau 1887 seine Allgemeine Elektrizitätsgesellschaft (AEG) gründete, stiegen Siemens und auch die Deutsche Bank als Kapitalgeber ein, so dass sie zusammen 75 Prozent der elektrotechnischen Produktion in Deutschland kontrollierten. ${ }^{10}$ Statt einer wettbewerbsorientierten >Marktwirtschaft< setzte sich eine Variante des Kapitalismus durch, die später gern »Deutschland AG« genannt wurde: Jeder war mit jedem verflochten, um lästige Konkurrenz gar nicht erst aufkommen zu lassen. Auch in anderen Leitbranchen wie der Chemie ging es ähnlich $\mathrm{zu}$.

Den wichtigen Großkonzernen ist es daher gelungen, den Markt seit mehr als 100 Jahren zu kontrollieren. Erhellend ist ein Blick auf den Börsenindex DAX, der die dreißig größten deutschen Aktiengesellschaften versammelt. Die Mehrzahl dieser Unternehmen wurde bereits vor dem Ersten Weltkrieg gegründet, und sie konnten sich bis heute behaupten, weil gegen ihre schiere Größe niemand mehr ankommt. Ob Stahl, Autos, Chemie oder Pharma: Diese Märkte sind weitgehend geschlossen und für Neulinge nicht mehr zu knacken.

Dieser Trend zur Konzentration erfasst auch neue Märkte, die durch technische Innovationen entstehen. Ein gutes Beispiel ist das Internet: Es dauerte jeweils weniger als zehn Jahre, bis Neugründungen wie Google, Facebook oder Amazon eine marktbeherrschende Stellung erreichten. Von echtem Wettbewerb ist auch das Internet weit entfernt, das einst als eine Zone der Freiheit gepriesen wurde.

Die Herrschaft der Großkonzerne ist selbst dem »Vater der sozialen Marktwirtschaft«, Ludwig Erhard, nicht gänzlich entgangen. In seinem berühmten Buch Wohlstand für alle (1957) beklagte er: »Die Entwicklung der modernen Technik förderte ihrerseits noch einmal gewisse Monopolisierungstendenzen, so dass ohne Zweifel die Gleichheit der Wettbewerbsbedingungen allenthalben störend beeinträchtigt wurde. « Allerdings war Erhard nicht bereit, sich zu fragen, was von seiner »Marktwirtschaft« noch übrig bleibt, wenn die wichtigen Branchen allesamt »Monopolisierungstendenzen« aufweisen.

Der moderne Kapitalismus ist eine Art Planwirtschaft - auch wenn sie der sozialistischen Planwirtschaft überhaupt nicht ähnelt. Natürlich ist es ein diametraler Unterschied, ob die Kalkulationen zentral in einem Ministerium oder dezentral bei privatwirtschaftlichen Firmen erfolgen. Aber geplant wird immer, weil geplant 
werden muss. Wenn das Risiko - und damit der Gewinn - nicht kalkulierbar wäre, würde überhaupt niemand investieren.

Unternehmen und Staat sind sich also viel ähnlicher, als gemeinhin gedacht wird. Dies räumt mit einem zweiten häufigen Missverständnis auf: Oft wird geglaubt, privates Unternehmertum und Staatsaktivitäten würden sich ausschließen. Doch Kapitalismus und Staat sind kein Gegensatz - sondern gemeinsam gewachsen.

Der Kapitalismus hätte sich ohne den Staat gar nicht entfalten können, denn es war notwendig, die Bevölkerung besser auszubilden, Universitäten zu gründen und Forschung zu finanzieren. Die explodierenden Städte mussten geplant und verwaltet, Straßen und Eisenbahnen gebaut werden. Potentiell gefährliche Medikamente mussten überwacht, die Sicherheit der Fabriken kontrolliert und Umweltschäden vermieden werden. Auch mussten Rentner/-innen abgesichert und Arbeitslose versorgt werden. Der Staat war plötzlich überall gefragt.

Zudem hätten zentrale technische Entwicklungen gar nicht stattfinden können, wenn der Staat nicht mitgezogen hätte. Um noch einmal auf die Firmengeschichte der AEG zurückzukommen: Für Emil Rathenau lohnte es sich nur, ins Elektrizitätsgeschäft einzusteigen, weil die Stadt Berlin als sicherer Kunde zur Verfügung stand und 1884 einen Konzessionsvertrag mit seiner Firma abschloss.

Die wachsende Bedeutung des Staates spiegelt sich in der sogenannten Staatsquote wieder, die den Anteil öffentlicher Ausgaben an der jährlichen Wirtschaftsleistung misst, und diese Staatsquote ist rasant gestiegen. Lag sie im Kaiserreich noch bei fünf bis sieben Prozent, hatte sie in der Weimarer Republik schon 15 bis 20 Prozent erreicht - und 2019 betrug sie in Deutschland 45,4 Prozent. ${ }^{12}$

Diese Entwicklung könnte die Vermutung nahelegen, dass die Staatsausgaben ständig steil nach oben klettern würden. Tatsächlich jedoch verharren sie seit 40 Jahren auf fast unverändertem Niveau. In Westdeutschland belief sich die Staatsquote 1975 auch schon auf 48,8 Prozent - und seither musste sogar noch eine Wiedervereinigung finanziert werden. Die Sorge ist also gänzlich unbegründet, dass ein Moloch namens Staat das angeblich zarte Pflänzchen namens Kapitalismus restlos zermalmen könnte.

Die hohe Staatsquote ist zudem keine Belastung für die Wirtschaft - sondern ihr Sicherheitsnetz. Denn die öffentlichen Ausgaben stabilisieren den Kapitalismus in Krisenzeiten: Renten laufen weiter, Arbeitslose werden unterstützt, und auch die Krankenkassen schränken ihre Leistungen nicht ein, wenn es zu einer Rezession kommt. Diese sogenannten rautomatischen Stabilisatoren garantieren einen Basissockel an Einkommen, was wiederum für Konsum, Umsatz und Arbeitsplätze

12 Bundesministerium der Finanzen: BMF Monatsbericht. April 2020, https://www.bundesfina nzministerium.de/Monatsberichte/2020/04/Inhalte/Kapitel-6-Statistiken/6-1-12-entwicklung -der-staatsquote.html (letzter Abruf 20.06.2020). 
sorgt, während die Firmen ihre Investitionen und Kapazitäten reduzieren. Würde die deutsche Wirtschaft nur aus privaten Unternehmen bestehen, würde sie in jeder Krise weitgehend kollabieren. Das 19. Jahrhundert ist dafür ein abschreckendes Beispiel, als es noch keine Sozialversicherungen gab und der Staat nicht intervenierte: Nach dem Gründerkrach 1873 wurden in der deutschen Eisenindustrie 40 Prozent aller Arbeiter entlassen. Die Löhne halbierten sich, und die Preise fielen um 38 Prozent. ${ }^{13}$ Dies wäre heute undenkbar.

Der Kapitalismus produziert Wachstum und damit Wohlstand, dennoch war im England des 19. Jahrhunderts nicht zu übersehen, dass das Proletariat verelendete. Hohe Löhne hatten die Industrialisierung in Großbritannien zwar ausgelöst, aber anschließend sank der Lebensstandard der Massen wieder, weil Menschen durch Maschinen ersetzt wurden. Die Volkswirtschaft als Ganzes wurde reicher, aber die Mehrheit der Bürger ärmer. Dieses Phänomen ist als »early growth paradox « in die Geschichtswissenschaft eingegangen. ${ }^{14}$

Eine Wende trat erst ab etwa 1880 ein, als die Gewerkschaften endlich legalisiert wurden und für höhere Löhne kämpfen konnten. Die steigende Kaufkraft der Massen hat den Kapitalismus bleibend verändert: Heute leben wir in einem Konsumkapitalismus, in dem die Nachfrage der Beschäftigten das gesamte System steuert.

Seither gilt im Kapitalismus ein ehernes Gesetz: Die Reallöhne müssen mit der Produktivität steigen. Nur wenn die Kaufkraft parallel zum technischen Fortschritt zunimmt, reicht die Nachfrage, um die wachsenden Gütermengen auch abzusetzen.

Sobald die Reallöhne stagnieren, gerät der Kapitalismus in Krisen. Lehrreich sind die USA nach dem Ersten Weltkrieg. Damals wurden die Reichen immer reicher, während die Beschäftigten von dem allgemeinen Wirtschaftswachstum nicht profitierten. Wie der französische Ökonom Emmanuel Saez berechnet hat, verfügte 1927 das reichste Zehntel der Amerikaner über 46 Prozent des USVolkseinkommens. Allein das oberste eine Prozent, also das reichste Hundertstel, monopolisierte bereits fast 24 Prozent der Wirtschaftsleistung. ${ }^{15}$

13 Vgl. Wehler, Hans-Ulrich: Deutsche Cesellschaftsgeschichte 1849-1914, München: C.H. Beck 1995, S. 552 ff.

14 Vgl. z.B. Jong, Herman de: „Living Standards in a Modernizing World - A Long-Run Perspective on Material Wellbeing and Human Development«, in: Wolfgang Clatzer et al. (Hg.), Clobal Handbook of Quality of Life. Exploration of Well-Being of Nations and Continents, Dordrecht u.a.: Springer 2015, S. 45-74, hier S. 58ff.

15 Vgl. Saez, Emmanuel: Striking it Richer: The Evolution of Top Incomes in the United States (Updated with 2013 Preliminary Estimates), WID.world TECHNICAL NOTE SERIES N²015/1. 
Prompt wurde es gefährlich: Die Produktivität in der Industrie hatte in den USA zwischen 1919 und 1929 pro Arbeiter um 43 Prozent zugelegt. ${ }^{16}$ Jeder einzelne Beschäftigte produzierte also fast 1,5-mal so viele Waren wie noch ein Jahrzehnt zuvor. Um eine Überproduktion zu vermeiden, hätten die Löhne entsprechend steigen müssen, damit die Arbeiter diese Flut an neuen Gütern auch hätten kaufen können. Doch da die Massenkaufkraft stagnierte, kam es schon bald zu einer Absatzkrise.

Spätestens ab 1927 gab es nur noch einen Ausweg, um doch noch, zumindest scheinbar, Gewinne zu generieren: die hemmungslose Spekulation. Doch diese Börsen-Hausse endete so furios, wie sie begonnen hatte. 1929 kam es zum »Schwarzen Freitag« an der Wall Street, und die Weltwirtschaftskrise begann.

Leider wiederholt sich diese Geschichte momentan: Erneut sind Einkommen und Vermögen extrem ungleich verteilt, wie der französische Ökonom Thomas Piketty in seinem internationalen Bestseller Das Kapital im 21. Jahrhundert anhand von aktuellen und historischen Steuerdaten nachgewiesen hat. ${ }^{17}$ Und erneut drohen Verwerfungen: Seit der Jahrtausendwende wurde Europa bereits von drei schweren Finanzkrisen heimgesucht, und es ist nur eine Frage der Zeit, bis eine vierte Finanzkrise ausbricht.

Dennoch wird der Kapitalismus nicht an seinen Finanzkrisen scheitern. Stattdessen ist er zum Untergang verdammt, weil er Wachstum benötigt, um stabil zu sein, aber in einer endlichen Welt unendliches Wachstum nicht möglich ist. Die Rohstoffe werden knapp, und zudem zerstört der Mensch seine eigenen Lebensgrundlagen, indem er die Umwelt verseucht. ${ }^{18}$

Der Kapitalismus wird chaotisch und brutal zusammenbrechen - nach allem, was man bisher weiß. Dieser Pessimismus mag zunächst übertrieben wirken. Schließlich fehlt es nicht an Konzepten, wie eine ökologische Kreislaufwirtschaft aussehen könnte, die den Kapitalismus überwinden soll. Einige Stichworte lauten: erneuerbare Energien, Recycling, langlebige Waren, öffentlicher Verkehr, weniger Fleisch essen, biologische Landwirtschaft und regionale Produkte. ${ }^{19}$

Doch das zentrale Problem ist leider ungelöst: Es fehlt die Brücke, die vom Kapitalismus in diese neue >Postwachstumsökonomie< führen soll. Über den Prozess der Transformation wird kaum nachgedacht. Der Kapitalismus fährt gegen eine Wand, aber niemand erforscht den Bremsweg.

16 Vgl. Galbraith, John Kenneth: The Great Crash 1929, Boston/New York: Mariner Books/Houghton Mifflin Harcourt 2009, S. 175.

17 Vgl. Piketty, Thomas: Das Kapital im 21. Jahrhundert, übersetzt von Ilse Utz und Stefan Lorenzer, München: C.H. Beck 2014.

18 Vgl. Bardi, Ugo: Der geplünderte Planet. Die Zukunft des Menschen im Zeitalter schwindender Ressourcen, übersetzt von Eva Leipprand et al., München: oekom 2013.

19 Vgl. etwa Klein, Naomi: Die Entscheidung Kapitalismus vs. Klima, übersetzt von Gabriele Gockel, Sonja Schuhmacher, Christa Prummer-Lehmair, Frankfurt a.M.: Fischer 2015. 
Die Vorschläge für eine Postwachstumsgesellschaft basieren immer auf der Idee, Arbeit und Einkommen zu reduzieren. Doch der Kapitalismus ist keine Badewanne, bei der man den Stöpsel ziehen und einfach die Hälfte des Wassers ablassen kann. Er ist kein stabiles System, das zum Gleichgewicht neigt und verlässliche Einkommen produziert, die man ruhig senken kann. Stattdessen ist der Kapitalismus ein permanenter Prozess. Sobald es kein Wachstum gibt, droht chaotisches Schrumpfen. ${ }^{20}$

Wie dieser Strudel funktioniert, hat der Schweizer Ökonom Hans Christoph Binswanger beschrieben, der unter anderem die Ökosteuer erfunden hat. Binswanger trieb die Frage um, ob der Kapitalismus auf das zerstörerische Wachstum verzichten könne. Seine Antwort lautete: Nein. Denn die »Investitionsketten« würden reißen, wie er es technisch ausdrückte. Übersetzt: Firmen investieren nur, wenn sie Gewinne erwarten. Gesamtwirtschaftlich sind diese Gewinne aber identisch mit Wachstum. Ohne Wachstum müssen die Unternehmen also Verluste fürchten. Sobald aber Profite ausbleiben, investieren die Unternehmen nicht mehr, und ohne Investitionen bricht die Wirtschaft zusammen. ${ }^{21}$ Es würde eine unkontrollierbare Abwärtsspirale einsetzen, die an die Weltwirtschaftskrise ab 1929 erinnert: Arbeitsplätze gehen verloren, die Nachfrage sinkt, die Produktion schrumpft, noch mehr Stellen verschwinden.

Nicht wenigen Wachstumskritiker/-innen ist diese systemische Sicht suspekt, die die Wirtschaft von >oben betrachtet. Sie würden lieber von >unten indem jede und jeder Einzelne seinen Konsum, aber auch seine Arbeitszusammenhänge verändert. Sie stellen sich die Wirtschaft als eine Summe vor, bei der viele kleine Nischen am Ende ein neues Ganzes ergeben. ${ }^{22}$

Doch damit machen die Wachstumskritiker/-innen den gleichen Fehler wie ihre neoliberalen Gegner/-innen: Sie glauben, dass die Wirtschaft nur eine Summe aller Unternehmen sei. Sie verwechseln Betriebs- mit Volkswirtschaft und verstehen nicht, dass der Kapitalismus ein Prozess ist, der Einkommen nur erzeugen kann, wenn es die Aussicht auf Wachstum gibt.

Da sich das Wachstum nicht einfach abschaffen lässt, machen neuerdings Konzepte wie »Green New Deal« oder »nachhaltiges Wachstum« Karriere. Sie wollen

20 Vgl. etwa Rifkin, Jeremy: Die Null-Wachstums-Cesellschaft. Das Internet der Dinge, kollaboratives Cemeingut und der Rückzug des Kapitalismus, übersetzt von Bernhard Schmid, Frankfurt a.M.: Campus 2014. Rifkin konzentriert sich auf den technologischen Wandel, kümmert sich aber nicht um die makroökonomischen Implikationen, die es hätte, falls seine These richtig wäre, dass im Kapitalismus keine Gewinne mehr zu generieren sind.

21 Vgl. Binswanger, Hans Christoph: Die Wachstumsspirale. Geld, Energie und Imagination in der Dynamik des Marktprozesses, Marburg: Metropolis 2006.

22 Vgl. etwa Paech, Niko: Befreiung vom Überfluss. Auf dem Weg in einer Postwachstumsökologie, München: oekom 2012. Oder: Felber, Christian: Die Cemeinwohl-Ökonomie. Das Wirtschaftsmodell der Zukunft, Wien/München: Deuticke 2010. 
Wachstum und Rohstoffverbrauch »entkoppeln«, indem die Effizienz gesteigert wird.

Diese »Entkoppelung« ist nicht völlig abwegig, denn seit 1970 hat sich der Energieverbrauch pro Wareneinheit halbiert. Die Umwelt wurde allerdings nicht entlastet, weil prompt der »Bumerang-Effekt « zuschlug. Die Kostenersparnis wurde genutzt, um die Warenproduktion auszudehnen, so dass der gesamte Energieverbrauch nicht stark fiel. ${ }^{23}$

Als Ausweg reicht es auch nicht, auf regenerative Energien umzustellen. Denn weite Bereiche der Wirtschaft lassen sich nicht mit Ökostrom betreiben. Das Elektroauto befindet sich noch immer im Versuchsstadium, und auch Passagierflugzeuge heben nur mit Kerosin ab. Allein der Flugverkehr zerstört aber jede Hoffnung, die Klimaziele zu erreichen, wie eine einfache Rechnung zeigt: Wenn die Erderwärmung begrenzt bleiben soll, darf jeder Mensch nur noch 2,7 Tonnen $\mathrm{CO}_{2}$ pro Jahr verursachen. Ein Flug von Frankfurt nach New York schlägt aber bereits mit 4,2 Tonnen zu Buche, und nach Sydney sind es gar 14,5 Tonnen. Damit wird wieder jener Verzicht unvermeidlich, der das Wachstum bedroht.

Es ist ein Dilemma: Ohne Wachstum geht es nicht, komplett grünes Wachstum gibt es nicht, und normales Wachstum führt unausweichlich in die ökologische Katastrophe. Es bleibt nur ein pragmatisches Trotzdem: trotzdem wenig fliegen, trotzdem Abfall vermeiden, trotzdem auf Wind und Sonne setzen, trotzdem biologische Landwirtschaft betreiben. Aber man sollte sich nicht einbilden, dass dies rein >grünes $<$ Wachstum sei. Wie man den Kapitalismus transformieren kann, ohne dass er chaotisch zusammenbricht - dies muss noch erforscht werden.

\section{Literatur}

AG Energiebilanzen e.V.: Energieverbrauch in Deutschland im Jahr 2018, Berlin: AGEB 2019.

Allen, Robert C.: Global Economic History. A Very Short Introduction, Oxford u.a.: Oxford University Press 2011.

Appleby, Joyce: The Relentless Revolution. A History of Capitalism, New York: Norton 2010.

Bardi, Ugo: Der geplünderte Planet. Die Zukunft des Menschen im Zeitalter schwindender Ressourcen, übersetzt von Eva Leipprand et al., München: oekom 2013.

Binswanger, Hans Christoph: Die Wachstumsspirale. Geld, Energie und Imagination in der Dynamik des Marktprozesses, Marburg: Metropolis 2006.

23 AC Energiebilanzen e.V.: Energieverbrauch in Deutschland im Jahr 2018, Berlin: ACEB 2019, S. 45 . 
Bundesministerium der Finanzen: BMF Monatsbericht. April 2020, https://www. bundesfinanzministerium.de/Monatsberichte/2020/04/Inhalte/Kapitel-6-Stat istiken/6-1-12-entwicklung-der-staatsquote.html (letzter Abruf 20.06.2020).

Erhard, Ludwig: Wohlstand für alle, Köln: Anaconda 2009.

Felber, Christian: Die Gemeinwohl-Ökonomie. Das Wirtschaftsmodell der Zukunft, Wien/München: Deuticke 2010.

Galbraith, John Kenneth: The Great Crash 1929, Boston/New York: Mariner Books/Houghton Mifflin Harcourt 2009.

Gall, Lothar: Die Deutsche Bank 1870-1955. 125 Jahre Deutsche Wirtschafts- und Finanzgeschichte, München: C.H. Beck 1995.

Jong, Herman de: »Living Standards in a Modernizing World - A Long-Run Perspective on Material Wellbeing and Human Development«, in: Wolfgang Glatzer et al. (Hg.), Global Handbook of Quality of Life. Exploration of WellBeing of Nations and Continents, Dordrecht u.a.: Springer 2015, S. 45-74.

Klein, Naomi: Die Entscheidung Kapitalismus vs. Klima, übersetzt von Gabriele Gockel, Sonja Schuhmacher, Christa Prummer-Lehmair, Frankfurt a.M.: Fischer 2015.

Osterhammel, Jürgen: Die Verwandlung der Welt. Eine Geschichte des 19. Jahrhunderts, München: C.H. Beck 2011.

Paech, Niko: Befreiung vom Überfluss. Auf dem Weg in einer Postwachstumsökologie, München: oekom 2012.

Piketty, Thomas: Das Kapital im 21. Jahrhundert, übersetzt von Ilse Utz und Stefan Lorenzer, München: C.H. Beck 2014.

Rifkin, Jeremy: Die Null-Wachstums-Gesellschaft. Das Internet der Dinge, kollaboratives Gemeingut und der Rückzug des Kapitalismus, übersetzt von Bernhard Schmid, Frankfurt a.M.: Campus 2014.

Saez, Emmanuel: Striking it Richer: The Evolution of Top Incomes in the United States (Updated with 2013 Preliminary Estimates), WID.world TECHNICAL NOTE SERIES $\mathrm{N}^{\circ} 2015 / 1$.

Schumpeter, Joseph A.: The Theory of Economic Development. An Inquiry into Profits, Capital, Credit, Interest and the Business Cycle, übersetzt von Redvers Opie, New Brunswick/London: Transaction Publishers 1983.

Sombart, Werner: Der moderne Kapitalismus. Erster Band: Die Genesis des Kapitalismus, Leipzig: Duncker \& Humblot 1902.

Statistisches Bundesamt (Destatis): Statistisches Jahrbuch 2018.

Vries, Peer: Escaping Poverty. The Origins of Modern Economic Growth, Wien: Vienna University Press/V\&R unipress 2013.

Wehler, Hans-Ulrich: Deutsche Gesellschaftsgeschichte 1849-1914, München: C.H. Beck 1995.

Ziegler, Dieter: Die industrielle Revolution, 3., bibliographisch aktualisierte Auflage, Darmstadt: Wissenschaftliche Buchgesellschaft 2009. 